\title{
Décharges capillaires pour la production de rayonnement X-mou cohérent et incohérent
}

\author{
D. Hong, C. Cachoncinlle, W. Rosenfeld, R. Dussart, N. Richard, R. Viladrosa, \\ J.M. Pouvesle et C. Fleurier
}

GREMI, UMR 6606 du CNRS, Université d'Orléans, BP. 6759, 45067 Orléans cedex 2, France

\begin{abstract}
Résumé : Après un aperçu des travaux antérieurs et actuels sur les décharges capillaires utilisées comme sources cohérentes ou incohérentes de photons X-mous, nous présentons deux expériences réalisées au GREMI. Dans la première expérience, la création d'un plasma d'argon fortement ionisé et de grande longueur ( $>60 \mathrm{~mm})$ conduit à un peuplement significatif du plasma en ions $\mathrm{Ar}^{7+}$ avec une énergie injectée de seulement $40 \mathrm{~J}$. Dans la seconde expérience, un plasma de carbone fortement ionisé est produit par ablation de la paroi d'un capillaire en polyéthylène. La température électronique de ce plasma est estimée à plus de $40 \mathrm{eV}$ à partir de la mesure de la résistivité du plasma. Les résultats actuels semblent montrer des amplifications d'émission spontanée (AES) sur la raie $\mathrm{H}_{\alpha} \mathrm{C}$ vi à $18.2 \mathrm{~nm}$ et sur la raie $\mathrm{H}_{\beta} \mathrm{C}$ vi à $13.5 \mathrm{~nm}$.
\end{abstract}

\section{INTRODUCTION}

Les sources compactes de forte brillance et de haut rendement énergétique, rayonnant dans le domaine des $\mathrm{X}$-mous intéressent particulièrement les industries de la microélectronique et des nanotechnologies pour la miniaturisation des motifs. D'autres applications importantes existent en biologie, en particulier pour les longueurs d'onde se trouvant dans «la fenêtre de l'eau» entre 2.3 et $4.4 \mathrm{~nm}$. De précédentes investigations [1-6] ont montré que la technique de décharge capillaire semble être très prometteuse pour la réalisation de telles sources. Soulignons que des amplifications d'émission spontanée (AES), appelé effet «laser X», ont été observées [1-2] dans ce type de décharge.

Une décharge capillaire est généralement réalisée en injectant une énergie de quelques dizaines de joules, emmagasinés dans un condensateur, dans un tube de très faible diamètre de l'ordre du millimètre et d'une longueur comprise entre quelques millimètres et quelques centimètres. $L$ 'inductance de ces systèmes doit être minimisée pour obtenir une forte impulsion de courant $I$ en un temps très court. Outre ces paramètres, le plasma dépend de la nature et de la pression du gaz utilisé et le cas échéant du matériau du capillaire employé. Dans ces décharges, la température électronique $T_{e}$ peut être supérieure à quelques dizaines d'eV pour une densité électronique $N_{e}$ de l'ordre de $10^{19} \mathrm{~cm}^{-3}[2-3]$.

Cette technique de production de plasma a un rendement énergétique élevé grâce au couplage direct entre l'énergie électrique stockée et le plasma. A partir d'impulsions de courant très courtes et compte tenu des dimensions caractéristiques des plasmas générés, elle -peut permettre d'obtenir des densités de puissance injectée extrêmement élevées. Enfin, la densité électronique étant très forte, le coefficient d'émission du plasma est également très fort. Ces trois qualités permettent d'assurer que les décharges capillaires ultra-rapides sont particulièrement adéquates pour la réalisation de sources de rayonnement dans un large domaine spectral s'étendant de l'infrarouge aux rayons X-mous.

Trois exemples d'utilisation de ces techniques comme sources incohérentes seront présentés dans le paragraphe suivant. Après une courte présentation concernant les résultats récents sur les lasers X pompés par décharges capillaires, nous abordons plus spécifiquement les développements réalisés au GREMI.

\section{DECHARGES CAPILLAIRES UTILISEES COMME SOURCES INCOHERENTES}

Les premiers travaux dans ce domaine remontent aux années soixante [4]. Avec une énergie stockée d'environ $40 \mathrm{~J}$, les auteurs avaient observé un fort continuum aux alentours de $434 \mathrm{~nm}$. La brillance 
spectrale $\beta_{\lambda}$ à $434 \mathrm{~nm}$ avait été mesurée à une valeur de $1.0 \times 10^{11} \mathrm{Wcm}^{-3} \mathrm{sr}^{-1}$, ce qui correspondait à un corps noir porté à une température de $450000 \mathrm{~K}$.

Très récemment, Juschkin et Kunze [5] ont produit un rayonnement intense dans 1'XUV vers $70 \mathrm{~nm}$ pour une énergie stockée de $240 \mathrm{~J}$. Dans cette expérience, les deux raies de la résonance de $\mathrm{Ar}$ VIII à 70.0 et $71.4 \mathrm{~nm}$ rayonnent comme un corps noir avec une énergie, pour chacune des deux raies, de $370 \mu \mathrm{J} \mathrm{sr}{ }^{-1}$ (2.6 $10^{I 2}$ photons) par impulsion. La durée assez longue de l'émission du rayonnement dans l'XUV, $700 \mathrm{~ns}$, est une des particularités de cette source.

Certaines applications requièrent des sources plus brèves. Une équipe du laboratoire LPMI à Palaiseau [6], étudie une autre famille de sources UV-X compactes et brillantes, par décharge capillaire dite ultrarapide. Cette décharge non-ablative utilise l'effet de cathode creuse pour créer un faisceau d'électrons favorisant le développement d'un plasma uniquement sur l'axe du capillaire. Avec une énergie stockée de seulement un joule, une intense émission XUV d'une durée d'environ 70 ns a été observée.

\section{DECHARGES CAPILLARES UTILISEES COMME SOURCES COHERENTES}

La théorie prévoit que l'effet laser est d'autant plus difficile à obtenir que la longueur d'onde désirée est plus courte. Dans le domaine des X-mous, cet effet n'a été obtenu qu'en 1985 [7], 25 ans après la découverte du laser. Dans ce travail précurseur, le milieu amplificateur était créé par l'impact d'un laser de grande puissance sur une feuille métallique de Sélénium. La cinétique de population du plasma chaud ainsi produit a conduit à une inversion de population et un effet d'AES pour les ions $\mathrm{Se}^{24+}$. Depuis lors, des progrès considérables ont été accomplis dans cette voie de pompage par laser, notamment pour tenter de réduire la taille des systèmes.

En 1988, J.J. Rocca et al. [8] ont proposé d'utiliser une décharge capillaire comme technique alternative pour obtenir un effet laser dans le domaine des rayons X-mous, avec les mêmes objectifs de réduire les dimensions du système et d'augmenter le rendement énergétique. La faisabilité de cette nouvelle technique a été clairement démontrée en 1994 par cette même équipe avec un produit gain-longueur de 7.2 pour une raie $\mathrm{d}^{\prime} \mathrm{Ar}^{8+}[1]$. Ce travail a ouvert une voie très prometteuse pour la création d'une source cohérente compacte facilement utilisable pour les applications. Jusqu'au début 97 , cette technique a permis d'obtenir l'effet laser X sur une dizaine transitions ioniques [9]. Depuis, des progrès importants ont été obtenus par l'équipe de J.J. Rocca, notamment l'effet laser sur la raie néonoïde de soufre à $60.8 \mathrm{~nm}$ [10] et le fonctionnement à $7 \mathrm{~Hz}$ du laser à $46.9 \mathrm{~nm}$ (argon néonoïde) avec une énergie moyenne de $80 \mu \mathrm{J}$ (énergie maximale : $125 \mu \mathrm{J}$ ) par impulsion [11].

\section{EXPERIENCES AU GREMI}

Deux expériences sur les décharges capillaires sont en cours au GREMI. La première est consacrée à l'étude du schéma de pompage par excitation collisionnelle [12] pouvant conduire à une AES sur la raie néonoïde d'argon Ar IX à $46.9 \mathrm{~nm}$. La seconde étudie le schéma de pompage par recombinaison collisionnelle [12] pouvant conduire à une $\mathrm{AES}$ sur la raie $\mathrm{H}_{\alpha} \mathrm{C}$ VI à $18.2 \mathrm{~nm}$.

Deux spectromètres XUV couplés à un détecteur MCP sans fenêtre sont utilisés pour les acquisitions des spectres des rayons $\mathrm{X}$-mous.

\subsection{Z-pinch dans l'Argon}

Le schéma de principe est donné figure 1. Deux condensateurs, de $40 \mathrm{nF}$ chacun et de très faible inductance, sont couplés directement au capillaire $(l=60 \mathrm{~mm}, \phi=4 \mathrm{~mm})$ qui se trouve au centre du montage. Un commutateur hors axe déclenche la décharge principale dans le capillaire rempli d'argon sous une pression d'environ 1 mbar. Le circuit électrique équivalent est donné figure 2 . Ce dispositif, dit Blumlein, a deux particularités intéressantes : d'une part, la différence de potentiel aux bornes du capillaire est 
initialement nulle bien que les condensateurs soient chargés à haute tension et, d'autre part, la densité de puissance injectée est très grande due au faible temps de montée $\tau$ du courant $(\tau<25 \mathrm{~ns}$ ). Cette rapidité du temps de montée du courant favorise la striction magnétique (auto-compression du plasma, dite l'effet $\mathrm{Z}$ pinch) nécessaire à la réalisation d'une cinétique conduisant à une inversion de population.

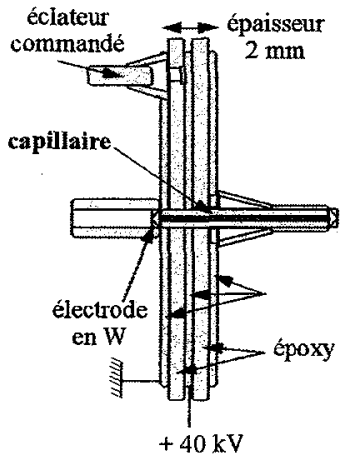

Figure 1 : Montage expérimental.

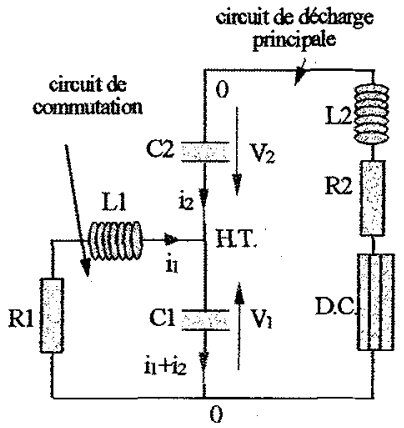

Figure 2 : Circuit électrique équivalent. Circuit Blumlein.

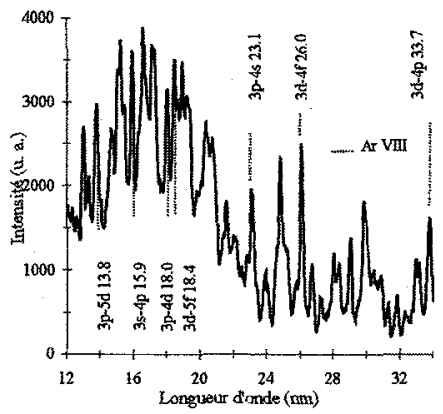

Figure 3 : Spectre d'argon dans le domaine $12-35 \mathrm{~nm}$

La figure 3 montre un spectre d'argon dans le domaine des rayons $\mathrm{X}$-mous. L'intensité des nombreuses raies identifiées de Ar VIII implique une forte population en ions $\mathrm{Ar}^{7+}$ durant la décharge. La présence d'ions de charge supérieure est très probable mais n'a pu encore être mise en évidence sur les spectres intégrés temporellement.

\subsection{Décharge ablative}

Différents capillaires en polyéthylène $\left(\left(\mathrm{CH}_{2}\right)_{\mathrm{n}}\right)$ ont été utilisés dans cette expérience. Leur longueur $l$ varie de 8 à $20 \mathrm{~mm}$ par pas de $2 \mathrm{~mm}$, tandis que le diamètre interne est de $1 \mathrm{~mm}$. Une pré-ionisation est nécessaire car le canal du capillaire est sous vide secondaire. Les 36 condensateurs de $2 \mathrm{nF}$ chacun, montés en parallèle, peuvent être chargés jusqu'à $-40 \mathrm{kV}$. Ceci correspond à une énergie stockée d'environ $60 \mathrm{~J}$. Le plasma est composé d'hydrogène et de carbone ablatés à la paroi. Le courant est mesuré à $26 \mathrm{kA}$ pour une tension de charge de $22 \mathrm{kV}$. En comparant le courant expérimental à celui calculé d'un circuit RLC série, l'inductance et la résistance moyennes peuvent être estimées à $L=30 \mathrm{nH}$ et $R=150 \mathrm{~m} \Omega$. Une résistivité typique de l'ordre de $10^{-3} \Omega \mathrm{cm}$ est également déduite du calcul pendant la première période. Avec une densité électronique supérieure à quelques $10^{17} \mathrm{~cm}^{-3}[2-3]$, on peut estimer la température électronique du plasma à plus de $40 \mathrm{eV}$, à partir de la formule donnée par Spitzer [13]. Cette forte température est confirmée par la présence de nombreuses raies de $\mathrm{C} \mathrm{V}$ et $\mathrm{C}$ VI sur le spectre dans le domaine des rayons $\mathrm{X}$-mous présenté figure 4 . Ces raies ont été identifiées à l'aide des tables de Kelly [14].

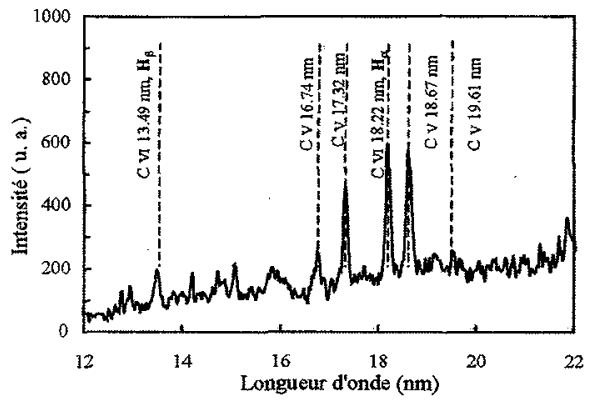

Figure $4: l=8 \mathrm{~mm}$, densité de puissance $=70 \mathrm{GW} / \mathrm{cm}^{3}$

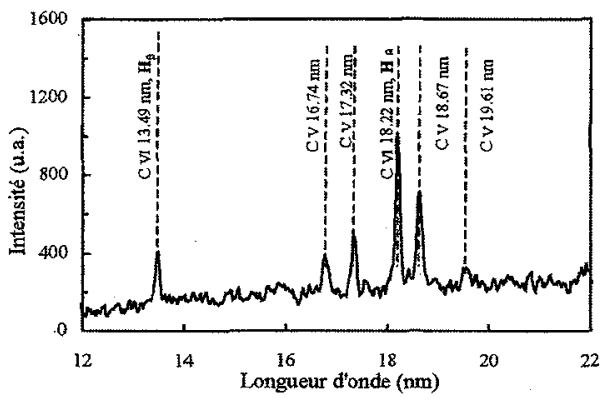

Figure $5: l=16 \mathrm{~mm}$, densité de puissance $=70 \mathrm{GW} / \mathrm{cm}^{3}$ 
Les spectres des figures 4 et 5 ont été obtenus avec la même densité de puissance pour deux longueurs de capillaire de 8 et $16 \mathrm{~mm}$. Pour le capillaire de $8 \mathrm{~mm}$, la raie $\mathrm{H}_{\alpha}$ de $\mathrm{C}$ VI à $18.2 \mathrm{~nm}$ a une intensité comparable à la raie C V à $18.6 \mathrm{~nm}$ (figure 4) alors qu'elle la domine largement pour un capillaire de $16 \mathrm{~mm}$ (figure 5).

La mesure de l'intensité des raies $\mathrm{C} V$ et $\mathrm{C}$ VI en fonction de la longueur du capillaire, à densité de puissance constante, montre une dépendance proche d'une exponentielle pour les raies $\mathrm{H}_{\alpha}$ et $\mathrm{H}_{\beta}$ de $\mathrm{C}$ VI (figures 6 et 7), alors que l'intensité des raies $\mathrm{C} V$ varie linéairement. Ceci semble montrer l'existence d'une AES sur ces deux transitions avec des gains de $1.6 \pm 0.4$ et $2.1 \pm 0.5 \mathrm{~cm}^{-1}$, respectivement. La raie $\mathrm{H}_{\beta}$ de $\mathrm{C}$ VI est particulièrement intéressante car sa longueur d'onde de $13.5 \mathrm{~nm}$ est très proche du pic de réflexion du miroir Molybdène - Silicium. Des mesures complémentaires à de plus grandes longueurs de capillaire sont nécessaires pour confirmer ces résultats prometteurs.

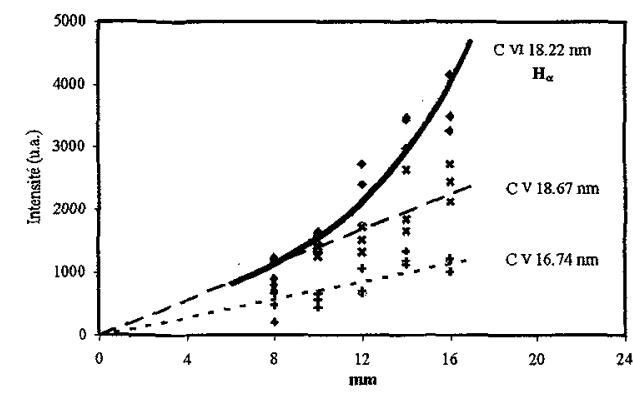

Figure $6:$ Intensité des raies en fonction de la longueur du capillaire avec la densité de puissance constante $\left(70 \mathrm{GW} / \mathrm{cm}^{3}\right)$

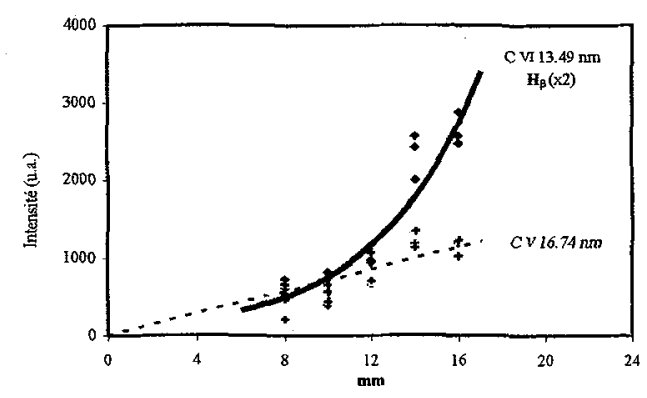

Figure 7 : Intensité des raies en fonction de la longueur du capillaire avec la densité de puissance constante $\left(70 \mathrm{GW} / \mathrm{cm}^{3}\right)$

\section{CONCLUSION}

Les décharges capillaires peuvent être utilisées comme sources incohérentes efficaces et pour la réalisation d'un futur laser X de "table". Les premiers résultats obtenus au GREMI sont très encourageants. En effet, pour l'expérience dite «Z-pinch dans l'argon», le circuit électrique retenu semble judicieux puisqu'il permet d'obtenir un fort courant ultra-rapide et un plasma à température très élevée. Pour l'expérience dite «décharge ablative», les résultats, à confirmer, semblent montrer une AES sur la raie $\mathrm{H}_{\alpha} \mathrm{C}$ VI à $18.2 \mathrm{~nm}$ et sur la raie $\mathrm{H}_{\beta} \mathrm{C}$ vi à $13.5 \mathrm{~nm}$.

\section{Références}

[1] J.J. Rocca et al., Phys. Rev. Lett. 73, (1994) 2192-2195

[2] H.-J. Shin et al., Phys. Rev. E 50 (1994) 1376-1382

[3] C.A. Morgan et al., Rev. E 49 (1994) 2282-2290

[4] P. Bogen et al., J. Opt. Soc. Am. 58 (2), (1968) 203-206

[5] L. Juschkin, H.-J. Kunze, Ruhr-Universität, Allemagne, communication privée.

[6] J. Larour et al., communication privée. P. Choi, M. Favre, Rev. Sci. Instrum, 69 (9), 3118, (1998).

[7] D.L. Matthews et al, Phys. Rev. Lett. 54 (1985) 110-113

[8] J.J. Rocca et al, Optics Lett. 13 (1988) 565-567

[9] J.M. Pouvesle, Ann. de Phys. (1997) C1-53-60

[10] F.G. Tomasel et al, Phys. Rev. A 55, (1997) 1437-1440

[11] B. R. Benware et al, Phys. Rev. Lett. 81, (1998) 5804-5807

[12] R.C. Elțon, X-ray Lasers, Academic Press, Boston MA, (1990)

[13] L. Spitzer, Interscience publishers, John Wiley\&Sons, New-York, London (1962), p143

[14] R. L. Kelly, J.Phys. Chem. Ref. Data, Vol 16, Suppl. 1, 1987 